unrelated-donor transplants were done. The cumulative incidence of acute GVHD of grade I-II and grade III-IV were $60 \%$ and $5 \%$, respectively. The myeloid donor chimerisms were all at least 94\% except one $86.1 \%$. Conservative treatment and HSCT decreased separately the frequency of infections to $5.1 \%$ and nearly 0 per 100 patient-months in patients.

Conclusions Severe infections were the main cause of death and the overall mortality was still high in China. HSTC is a potentially curative therapy for CGD leading resolution of infections and complications. CGD children who had undergone HSCT have better quality of life and fewer infections compared with those treated conservatively.

\section{GP194 PERIODIC FEVER-THE IRISH PFAPA STORY SO FAR}

${ }^{1}$ Ana Louise Hawke*, 'Timothy Ronan Leahy, ${ }^{1}$ Jayne M MacMahon, ${ }^{1} E m m a$ Jane MacDermott, ${ }^{2}$ Karina Butler, 'Patrick Gavin, 'Orla G Killeen. 'Our Lady's Children's Hospital, Crumlin, Dublin, Ireland; 'Our Lady's Children's Hospital, Crumin, Dublin, Ireland

\subsection{6/archdischild-2019-epa.254}

Introduction Periodic fever, aphthous stomatitis, pharyngitis and cervical adenitis (PFAPA) syndrome is the most common autoinflammatory disorder in childhood, with multifactorial, polygenic causes postulated.

Objective To appraise the clinical features, inflammatory characteristics and management of children with PFAPA attending a tertiary Autoinflammatory Clinic.

Methods A retrospective observational chart review of all children with confirmed clinical or suspected PFAPA attending the autoinflammatory clinic at Our Lady's Children's Hospital, Dublin from January 2016.

Data were collected on basic demographics, route of referral, symptoms and signs and inflammatory markers during disease episodes (febrile) and non-episodes. Molecular gene analysis if performed, were included. Documentation of all therapeutic agents to date was collated.

Results Thirteen children were identified as having PFAPA.

The median age of disease onset was 16 months, (4 months to 4 years). The route of referral was via Immunology (4 patients), Rheumatology (6 patients) and Infectious disease (3 patients).

All children presented with episodic, recurrent febrile episodes with associated features. (table 1). Median range of duration of episodes was 3-4 days.

\begin{tabular}{|c|c|c|c|c|c|}
\hline \multirow[t]{7}{*}{ Associated Symptoms: } & \multicolumn{2}{|l|}{ Stomatitis } & \multicolumn{3}{|l|}{8} \\
\hline & \multicolumn{2}{|c|}{ Tonsillitis/Pharyngitis } & 8 & & \\
\hline & \multicolumn{2}{|c|}{ Cervical Adenitis } & 7 & & \\
\hline & \multicolumn{2}{|c|}{ Lethargy } & 6 & & \\
\hline & \multicolumn{2}{|l|}{ Rash } & 4 & & \\
\hline & \multicolumn{2}{|l|}{ Anorexia } & 4 & & \\
\hline & \multicolumn{2}{|l|}{ GI upset } & 12 & & \\
\hline Periodicity of disease & $<2$ weeks & 1 patient & Flare & $2-3$ days & 5 patients \\
\hline flare & 2-4 weeks & 10 patients & Duration & $4-5$ days & 7 patients \\
\hline & 4-6 weeks & 2 patients & & $6-7$ days & 1 patient \\
\hline
\end{tabular}

$69 \%$ of patients had documented raised inflammatory markers during a flare, with $84 \%$ having high serum amyloid
A (SAA) levels, the highest documented being $1220 \mathrm{mg} / \mathrm{l}$ (< 10 normal). Genetic testing in 6 children was negative for other causes of hereditary autoinflammatory disorders.

11 patients had a significant response to an initial trial of corticosteroids, 2 reported rebound flares. Colchicine was the treatment of choice (11), 9 who had a good response. Tonsillectomy was performed in 5 patients, 3 of whom reported benefit. Biologic agents, Anakinra (2) and Adalimumab (1) were instituted in those refractory to colchicine with variable response.

Conclusion This study gives an over view of the burden of disease imposed by PFAPA on an Irish population. All the children presented with fevers, not all had the triad of aphthous stomatitis, pharyngitis and cervical adenitis.

The majority of patients had relief of symptoms with an initiation trial of corticosteroid. Colchicine was the most frequently used therapeutic agent to prevent disease flares.

Tonsillectomy and biological agents are potential alternative options in some resistant/severe cases.

\section{GP195 ANTIBIOTIC THERAPY IN CHILDREN HOSPITALIZED DUE TO RSV LOWER RESPIRATORY TRACT INFECTION}

1,2Małgorzata Czajkowska* 1,2 August Wrotek, 1,2Teresa Jackowska. 'Department of Pediatrics, the Medical Centre of Postgraduate Education, Marymoncka 99/103, 01-813,

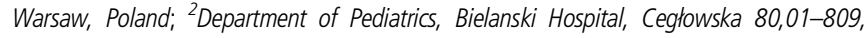
Warsaw, Poland

\subsection{6/archdischild-2019-epa.255}

Background Polish guidelines do not support antibiotic therapy in children with RSV (Respiratory Syncytial Virus) infection with few exceptions. We analyzed the frequency and reasons for antibiotic treatment in children under 2 years of age to assess the most important drivers in antibiotic use, and its correlation with routinely used inflammatory markers.

Material and Methods 198 children (median age3 months) were hospitalized due to RSV lower respiratory tract infection (RSV-LRTI) in two consecutive seasons (2016/2017 and 201718). RSV infection was confirmed with rapid antigen test and/or polymerase chain reaction. 197 children were enrolled into the study.

Results Antibiotics were used in 39\% (76/197) patients, including 6 patients $(8 \%)$ who received broad-spectrum antibiotics. The main reason for antibiotic use was pneumonia $(57 \% ; 43 /$ 76), followed by acute otitis media (AOM) in 18\% (14/76), lack of improvement $(15 \% ; 11 / 76)$, and urinary tract infections (UTI) in $10 \%(8 / 76)$. Children who received antibiotics were older (median 3.5 vs. 2 months; p<0.01), presented with statistically higher C-Reactive Protein $(6.65$ vs. $2.1 \mathrm{mg} / \mathrm{L}$; $\mathrm{p}<0.01)$, andprocalcitonin (PCT) level $(0.13$ vs. $0.09 \mathrm{ng} / \mathrm{mL}$; $\mathrm{p}<0.01)$, white blood cells $\left(12\right.$ vs. $10.6 \times 10^{\wedge} 3$ cell $/ \mathrm{uL}$; $\mathrm{p}=\mathbf{0 . 0 4})$ and absolute neutrophil count (4.09 versus $2.17 \times 10^{\wedge} 3$ cell $\left./ \mathrm{uL} ; \mathbf{p}<0.01\right)$. The differences had no clinical significance. Patients were further analyzed in the subgroups: pneumonia, AOM, lack of improvement and UTI group, but no clinically relevant differences were found. Only patients who were given broad-spectrum antibiotics had clinically and statistically significance in PCT (1.95 vs. $0.1 \mathrm{ng} / \mathrm{mL} ; \mathrm{p}=0.01)$. AOM in older children were at 3.96-fold increased risk of antibiotic therapy (95\%CI: 1.1-14.7). Patients who received antibiotics required longer hospital stay (11.5 vs. 9 days, $\mathrm{p}<\mathbf{0 . 0 1}$ ), especially when broad-spectrum antibiotics were given (17 versus 9 days, $\mathrm{p}<0.01$ ). 